\title{
Whitewater recreationists' preferences for social, resource and managerial attrib- utes in the Alpine Nature and Geopark Styrian Eisenwurzen
}

\author{
Silvia Kainzinger, Arne Arnberger \& Robert C. Burns
}

Keywords: whitewater recreation, crowding, preferences, discrete choice experiment

\section{Abstract}

The Salza River is located in the Alpine Nature and Geopark Styrian Eisenwurzen and is used heavily for whitewater recreation. One of the main goals of protected area management is to provide opportunities for outdoor recreation to maintain or enhance the quality of visitor experience. This study explored whitewater recreationists' crowding perceptions and preferences for river trip scenarios on the Salza River, Austria, using a discrete choice experiment. Six attributes described social, resource and managerial river conditions. More than $70 \%$ of the paddlers reported crowding. Results of the discrete choice experiment suggest that use levels are by far the most important attribute, followed by the river difficulty and the river access fee. Boaters preferred low numbers of people on the river, no waiting times for car parking or boat launching, moderate river difficulty, shorter trip lengths and a low river access fee. The findings could be of use to the Nature Park in helping to better understand the preferences of one of its main target groups.
Profile

Protected area

Alpine Nature and Geopark Styrian Eisenwurzen

Mountain range

Alps, Austria

\section{Introduction}

Several visitor management frameworks, employed mainly in protected areas (PA) in the United States, are based on the concept of carrying capacity and the associated indicators and standards of quality. Carrying capacity addresses how much use can be accommodated without unacceptable impacts to park resources or the quality of visitor experiences (Manning 2013). Examples of management approaches that determine indicators and standards of quality in parks and PAs include the Limits of Acceptable Change (LAC) (Stankey et al. 1985) and the Visitor Experience Resource Protection (VERP) (National Park Service 1997).

If use levels exceed preferred site conditions, visitors will employ coping behaviours such as intra- or inter-area use displacement. When coping behaviours fail, they will perceive crowding, which diminishes the quality of the outdoor experience (Arnberger \& Eder 2012; Heberlein \& Vaske 1977; Manning 2013). Therefore, surveys on crowding perceptions and preferred conditions of visitors at recreation sites are a fundamental responsibility of PA managers.

Outdoor recreation opportunities are generally recognized as having three dimensions - social, resource and managerial (Manning 2011). Social attributes (levels and types of use) address recreational use; resource conditions (e.g. landscape, topography) are associated with natural qualities, and managerial components (e.g. use regulations) are determined by the management of the resource (Clark \& Stankey 1979). PA managers are required to make trade-offs among these three conditions in their daily management, and visitors also balance several conditions when choosing their preferred recreation site (Van Riper et al. 2011).
One management framework used to define diversity in recreation experiences is the Recreation Opportunity Spectrum (ROS) (Clark \& Stankey 1979). The framework recognizes that a recreation experience is related to the settings in which the activity occurs, and the settings are in turn a function of social, resource and managerial attributes (Clark \& Stankey 1979).

While there is some literature concerning preferences and trade-offs for social, resource and managerial attributes, the predominant focus of past work has been on PAs in the U.S. (Lawson \& Manning 2002; Bullock \& Lawson 2008; Van Riper et al. 2011). River recreation is an unknown quantity in Austria (Chiari et al. 2008), although several rivers are heavily used, potentially leading to crowding, user conflicts and conflicts between nature conservation and recreation. However, information is lacking on river recreationists and their perceptions of and preferences for social, resource and managerial river conditions. This study explores crowding perceptions, and preferences for and tradeoffs among social, resource and managerial attributes of whitewater boaters on the Salza River, Austria.

\section{Boaters' perceptions and preferences}

Many studies focus on social impacts of too much recreational use of a river because crowding can have a negative impact on boaters' trip satisfaction (Herrick \& McDonalds 1992; Whisman \& Hollenhorst 1998). Research often addresses the perception of crowding, user conflicts, and preferences for encounters with other river users at various river sites, as well as waiting times at rapids or at boat ramps. Crowding perceptions of whitewater boaters vary according to the location on the river, with kayakers and rafters being most concerned about crowding at the rapids (Tarrant et al. 
1997). Kainzinger et al. (2016) compared perceived crowding in two different river settings using a 9-point crowding scale. Boaters on a high-use river reported higher crowding levels $($ mean $=3.72)$ than paddlers from a low-use setting (mean =1.89) (Kainzinger et al. 2016).

There are situations, however, where higher densities have been evaluated positively (Anderson et al. 1998), and even contributed to higher satisfaction of river recreationists (Ditton et al. 1983) or hunters (Heberlein et al. 1982). These results lead to the conclusion that a scale measuring the negative evaluation of $a$ certain density or number of encounters may not always adequately capture the perception of there being an insufficient number of people (to give a sense of security, for example), and as a result does not always record optimal conditions (Arnberger \& Mann 2008). In our approach, we decided to follow the recommendation of Vaske and Shelby (2008) to continue the use of the single-item crowding scale for the sake of future meta-analyses.

Past research on boaters' preferences revealed that paddlers generally prefer lower use levels (Kainzinger et al. 2016; Shelby \& Heberlein 1986; Tarrant et al. 1997) and encounters with the same user group as their own, while encounters with other groups are least preferred at rapids (Tarrant et al. 1997). Boaters' preferences for use levels on the river differ between low- and high-use settings, suggesting that they specifically choose a setting based on their preferences (Kainzinger et al. 2016).

Boaters reported their trip enjoyment was decreased when waiting times at the rapids occurred (Stewart et al. 2000). Whittaker and Shelby (1988) observed that day users were more concerned about having to wait at the boat ramp, while Kainzinger et al. (2016) found waiting times at the boat ramp and for parking did not concern boaters. Resource factors such as the difficulty of the river section could increase boaters' enjoyment, and are often a reason why boaters visit a river (Herrick \& McDonalds 1992; Kainzinger et al. 2016). Whitewater boaters were motivated by challenge (Galloway 2012) and more experienced boaters sought more difficult whitewater (Lee et al. 2007). Boaters on day trips preferred a trip length of four hours, while eight hours is disliked (Kainzinger et al. 2016).

In North America, use restrictions and river-use allocation systems are used on about 110 rivers to protect the natural resource and the quality of the visitor experience (Shelby et al. 1989a; Siderelis \& Moore 2006; Whittaker \& Shelby 2008). In Austria, commercial outfitter companies often need to have a permit for river trips. In Styria, for example, permits are required for boats that take more than three people (Raftingverband Steiermark 2016). Kainzinger et al. (2016) found that paddlers who frequently participate in whitewater activities dislike a river access fee of $\$ 8$, although the fee was not very important for them in deciding to visit a river or not.

\section{Managing whitewater recreation in PAs}

For PAs, scenic quality, as well as ecological, social, educational and managerial aspects have to be integrated in the management strategy (Manning \& Anderson 2012). Limiting use may not be enough to achieve resource protection. A well-known example is the management plan for the Colorado River in the Grand Canyon National Park, developed by the U.S. National Park Service. This plan relies on the twin strategies of limiting the use and reducing the impact of use (Grand Canyon National Park 2006), implemented by using a coordinated suite of management practices, such as spatial and temporal zoning of the river, regulations of the number and type of boating trips (regulations and allocation), ranger patrols to enforce regulations, a lottery system to allocate permits to non-commercial boaters, and an intensive programme of public education (Manning \& Anderson 2012). In Austria, whitewater recreation in a PA occurs, for example, on the Enns River, located in the Gesäuse National Park. Access to the river is permitted only at designated sites, and walking on the river banks is prohibited. In specific areas, interpretive signs present information about the sensitive river ecosystem and endangered species. During peak season, there are onsite environmental education programmes led by park rangers (Chiari 2010).

\section{Study aims}

The Salza River, located in a Nature Park in Austria, is used greatly for whitewater recreation. Whitewater kayakers usually paddle in groups with other kayakers of a similar level of competence (Schuett 1995), use similar rivers, and are typically motivated by challenge (Galloway 2012). To define the preferences of the target group of whitewater boaters, this study investigated their crowding perceptions, and preferences for and trade-offs among social, resource and managerial attributes of a recreational river, using a discrete choice experiment.

Discrete choice experiments have frequently been applied to explore preferences for and trade-offs among recreation conditions (Arnberger \& Eder 2015; Bullock \& Lawson 2008; Kainzinger et al. 2016; Louviere \& Timmermans 1990; Van Riper et al. 2011). Unlike conventional univariate preference studies, this method allows the analysis of recreationist trade-off behaviour among river recreation-related factors, thus offering a more realistic portrayal of human behaviour (Lawson \& Manning 2002; Louviere et al. 2000). In a choice experiment, respondents are asked to simultaneously evaluate multiple alternatives of hypothetical, multi-attribute, goods or services (Louviere et al. 2000). Such alternatives (e.g. river trip scenarios) are defined as combinations of river attributes (Hensher et al. 2005). In a discrete choice experiment, two or more hypothetical alternatives are combined into choice sets and respondents choose the most and / or least preferred river scenario from each set they are 


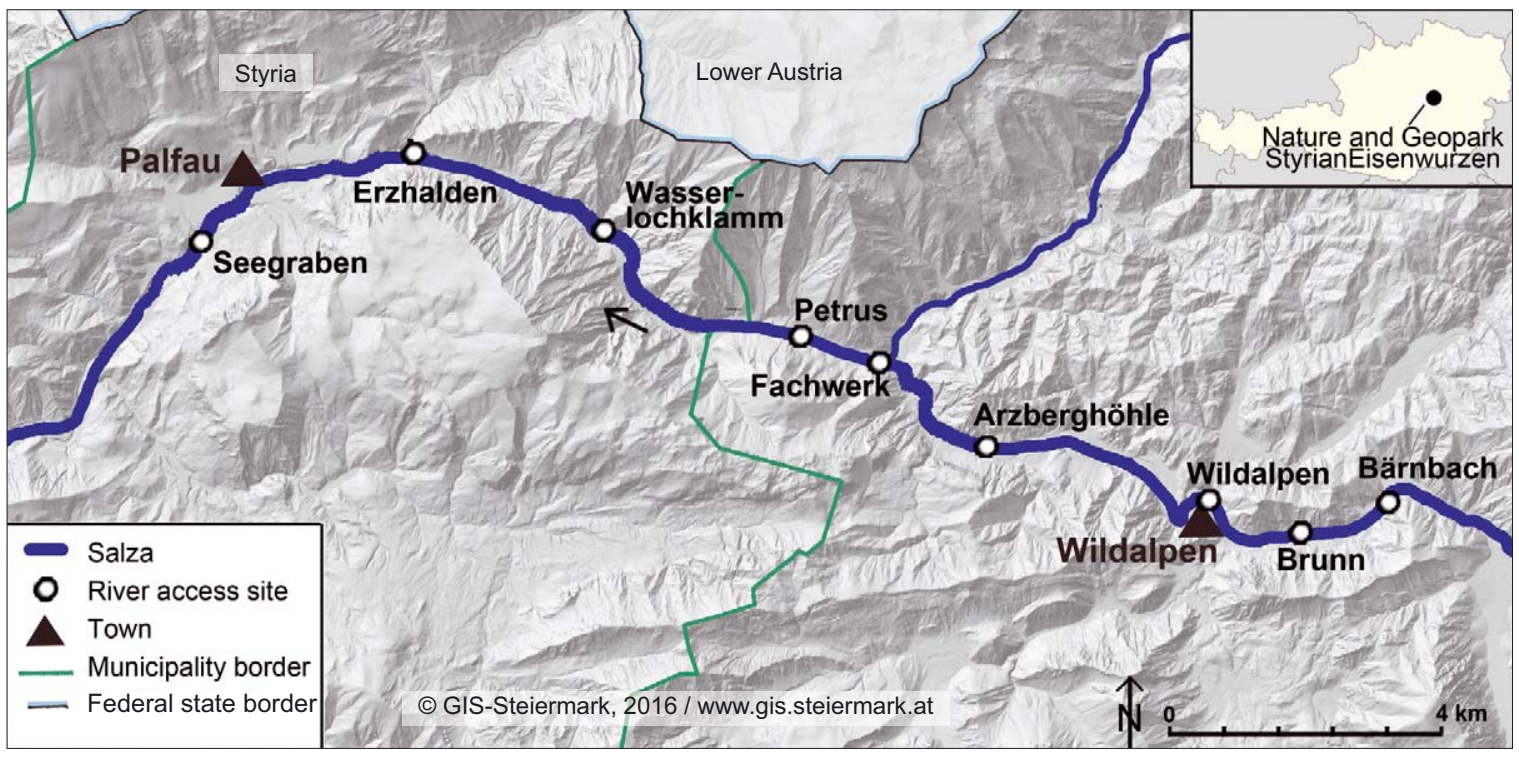

Figure 1 - Area map of the Salza River between the towns of Wildalpen and Palfau.

asked to evaluate. This method is rooted in the traditional micro-economic theory of consumer behaviour and preference theory. Random utility theory (Louviere et al. 2000) suggests that choices can be modelled as a function of the factors of the alternatives. Selection of one alternative over the other implies that the utility of that alternative is greater than the utility of the other.

We developed the following research questions to guide this study:

R1: Do boaters on the Salza River feel crowded?

R2: What are boaters' preferences for the number of people on the river, waiting times for boat launch and for parking? (Social attributes)

R3: What are boaters' preferences for river difficulty and trip length? (Resource attributes)

R4: What are boaters' preferences for river access fees? (Managerial attributes)

R5: Does trade-off behaviour among social, resource and managerial attributes exist? (Relative importance)

\section{Methodology}

\section{Study area}

Data on river users were collected on the Salza River, located in the province of Styria in Austria (Figure 1). The Salza is part of the Alpine Nature and Geopark Styrian Eisenwurzen. The Salza is a popular location for whitewater recreation, with class-I to class-III rapids along 35 kilometers of its length. Between the towns of Wildalpen (a designated Nature Park town) and Palfau, the river is in some areas very narrow. A popular whitewater play spot for boaters is located next to the Wasserlochklamm. A 1992 amendment to Styrian state law restricts Salza River recreational rafting to groups of three or less per boat. Only registered, commercial companies with certified guides are permitted to conduct rafting tours with more than three people per boat from April $25^{\text {th }}$ to October $15^{\text {th }}$ each year. Kayaks and canoes are permitted regardless of the group size. Interpretive signs located along the river provide information about river access sites and river use restrictions. This information is provided in eight different languages, as use from whitewater recreationists from neighbouring countries such as Czech Republic has increased over the past few years (Raftingverband Steiermark 2016).

\section{Data sampling}

A total of 340 interviews were collected from May to September 2014, with a response rate of $84 \%$. We approached respondents at two campsites in the town of Wildalpen, after their whitewater trip was over for the day. The surveys were self-administered and the interviewer asked the paddlers to fill out the questionnaire on site. Everyone who was willing to participate in the survey was handed a questionnaire. German and English versions of the questionnaire were used. If a person was able to understand neither the German nor the English questionnaire, they were excluded from the study (this process excluded fewer than ten people during the survey period). To avoid coverage error, the sample was stratified over weekdays and weekend days, spread across subgroups, and conducted on site (Vaske 2008). As no information on recreational river use exists so far, we cannot definitely say whether our sample is representative. However, we believe that our sample is representative for overnight river boaters because of the high response rate and survey design. Our study was limited to private boaters only, who were not using the service of a guide for their river trip. Sixteen questionnaires were eliminated because of incomplete data. 


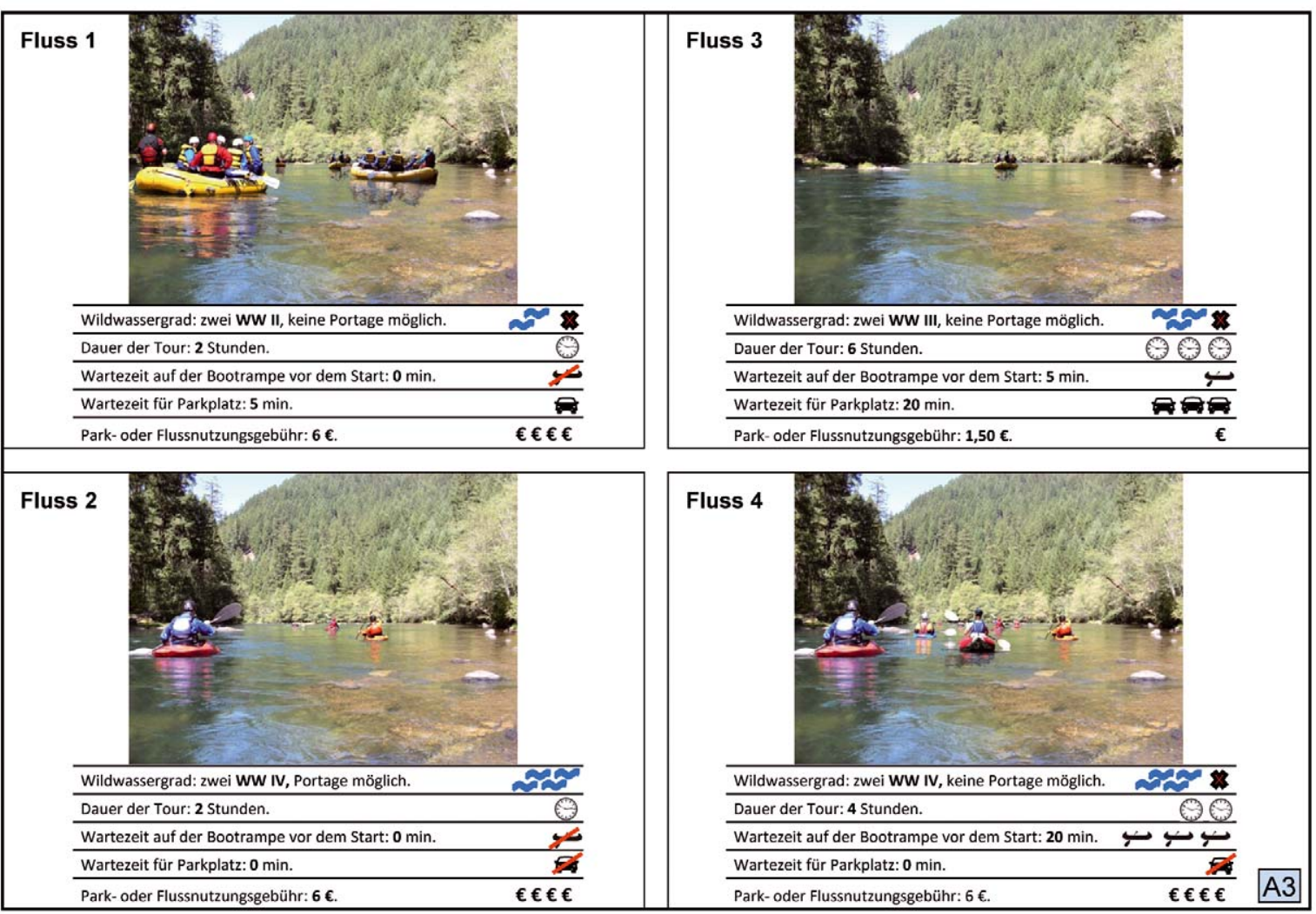

Figure 2 - Example of a stated choice set. The respondents had to select their most and least preferred options out of four river scenarios based on the attribute levels.

\section{Questionnaire}

The survey contained questions about socio-demographic characteristics and visit-related questions, such as perceived and expected crowding and waiting times for car parking, boat launch and boat take-out from the water. Perceived crowding was measured using a single-item, 9-point scale (Heberlein \& Vaske 1977). We decided to use the single-item crowding measure as it has been widely applied in outdoor recreation research, particularly in previous studies on whitewater recreation (for literature review, see Vaske \& Shelby 2008), and because of the ease in interpreting results. Additionally, we asked the respondents to indicate the percentage of time spent in sight of other groups, and the acceptable percentage of time spent in sight of them. Boaters rated their personal skill level on a 5-point scale (beginner, basic, intermediate, advanced, expert), and the difficulty of rapid class which they felt comfortable tackling by themselves, ranging from class I to class V (Bricker \& Kerstetter 2000). Each respondent was shown a set of river trip scenarios to assess their river preferences (see Figure 2).

\section{Choice experiment}

The study used a choice survey with visual and verbal presentations of six variables. Conditions were displayed using 128 riverscape scenarios, organized into 32 choice sets. Each individual evaluated four choice sets, each containing four scenarios. The interviewee chose the most and the least preferred river trip out of the four scenarios (Arnberger \& Haider 2005). The choice sets were systematically rotated to avoid starting-point bias.

The scenarios consisted of the systematic presentation of six attributes (one photograph and a list of five elements for consideration) (Figure 2). The attributes selected were found in previous research to be generally applicable for whitewater recreation (Kainzinger et al. 2016; Lee et al. 2007; Manning et al. 1995). Three attributes related to social conditions (i.e. number of people on the river, waiting time for boat launch, waiting time for parking), two factors captured resource river conditions (river difficulty, trip length), and one addressed managerial attributes (river access fee).

The social attribute number of people on the river was based on the density measure PAOT (persons at one time) (Manning et al. 1995) and presented different use levels and user types on the river (1, 4, 8 or 12 kayakers; 1 , 4 or 8 rafters; 6 kayaks and 6 rafts). We assumed that a raft was carrying six people. Two social attributes (waiting times for boat launch and waiting time for parking) addressed facility-related congestion. These two attributes were rated on a 4-level scale for waiting times of 0 to 20 minutes. This paper is part of a larger study, and the variables were based on the conditions of a lowuse river (North Umpqua River) in the U.S., where we did not see waiting times before rapids or for portage as a major problem (Kainzinger et al. 2016). However, since waiting-time issues before rapids might be relevant for the Salza River (a river with high recreational 


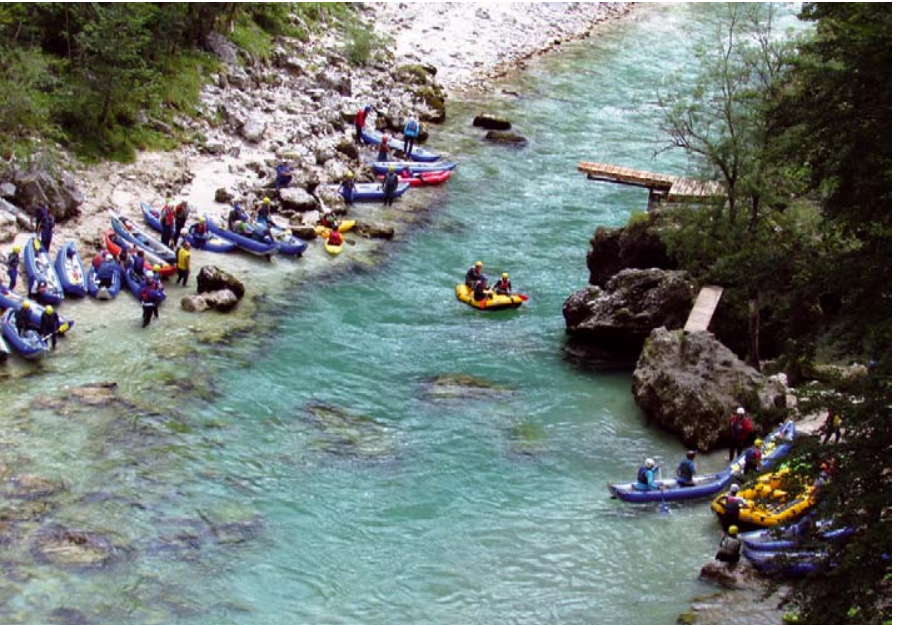

Figure 3 - Boaters at the play spot close to the Wasserlochklamm. (C) S. Kainzinger

use), it might be considered a limitation of the present study not to have included this variable.

The resource attributes addressed one of the main motivations of whitewater boaters, the search for challenge (Galloway 2012) (river difficulty), and their physical condition, using the resource length on the river (trip length) as a measure. The 4-level attribute river difficulty ranged from two class-II to two class-IV rapids with and without portage. Trip length ranged from two to eight hours. We assumed that a very short river trip (less than two hours) might not be worth the effort of travelling to the whitewater location. On the other hand, if the river section is too long (more than eight hours), it might not be physically achievable for some boaters (Kainzinger et al. 2016). The managerial attribute river access fee is based on the fact that river use is often managed using a permit system. River access fee represents boaters' willingness to pay for the desired river condition. The 4-level attribute ranged from 1.50 to $6 €$ per person.

One social attribute, number of people on the river, was displayed with digitally calibrated images showing different use levels and user types on the river. The picture of the riverscape showed a site on the North Umpqua River taken at eye level on a sunny day, and was designed using Photoshop Version CS4. The remaining five attributes were presented using verbal descriptions and pictograms. Digitally calibrated images have frequently been used to study, for example, trail preferences of visitors to urban forests (Arnberger \& Haider 2005; Arnberger \& Eder 2015) or beach preferences of whitewater river users (Stewart et al. 2003). The increasing application of visual research methods exposes a number of challenges that might influence the utility of this approach. These include the validity and reliability of visual measures (Palmer \& Hoffman 2001), the degree of realism of simulated environments, and the ability of static images to fully capture experiential sensations (Reichhart \& Arnberger 2010). However, much of the past literature in outdoor rec- reation using visual research approaches has found this method to be relatively robust and useful (e.g. Arnberger \& Haider 2005; Arnberger \& Eder 2015; Manning et al. 1996; Van Riper et al. 2011).

\section{Data analyses}

A paired sample t-test was conducted to test for differences between acceptable percentage of time in sight of other groups and actual percentage of time spent in their sight. Following the approach suggested by Shelby et al. (1989b), we split the 9-point crowding scale into two groups. The first group ranged from 1 to 2 , indicating no crowding; the second one concerned situations where boaters reported some level of crowding (scale points 3 to 9).

To analyse boaters' preferences, maximum likelihood analysis was performed with Latent GOLD Choice 4.0. As this study integrated six attributes in one design, each parameter estimate (part-worth utility) of the multinomial logit model indicates the magnitude of difference of this attribute level from the overall mean of all attribute levels (Hensher et al. 2005; Louviere et al. 2000). Therefore, the estimates are all relative to each other. No base alternative or no-choice alternatives were presented and, therefore, no intercept exists. McFadden's rho square $\left(r h o^{2}\right)$ was used to indicate the goodness of fit of the estimated choice models. Values of $r h o^{2}$ between 0.2 and 0.4 are considered to be indicative of extremely good model fits (Louviere et al. 2000). The fit for the model in this study was acceptable, with a $r h o^{2}=0.07$. We calculated the relative importance of each attribute on riverscape choices following the approach developed by Vermunt and Magidson (2003). Additional multivariate models were developed testing for differences between the country of origin (Austria, Germany, Czech Republic) and for different levels of crowding perceptions, but the models did not reveal any significant differences.

\section{Results}

\section{Sample profile}

The majority of the boaters on the Salza River were from Austria (41.2\%), Germany (34.0\%) and Czech Republic (23.2\%). Most of the paddlers (76.9\%) were repeat visitors and had been coming to the river, on average, for 14 years. On average, boaters spent 9.78 days per year on the Salza and 36.27 days per year on other rivers $(t=-7.033, p<.001)$. Boaters rated themselves as intermediate $($ mean $=3.18)$ and able to run at least a class-III rapid (mean $=3.21)$.

\section{Crowding Indicators}

Boaters felt slightly crowded while on the river (mean $=4.05$ ), and $71 \%$ of the paddlers reported crowding. Paddlers were $35 \%$ of their time in sight of other groups, which is less than the acceptable $42 \%$ of time $(t=-4.07, p<.001)$. On average, boaters waited 
less than a minute for parking, 1.4 minutes at the boat launch, and less than a minute at the take-out.

\section{River preferences}

All attributes predicted whitewater boaters' choices. The results of the social attribute number of people on the river revealed that boaters prefer fewer than six people per boat. The presence of more than eight people was perceived negatively. Paddlers disliked waiting times of more than five minutes for boat launch and parking. For the resource attribute river difficulty, the level with two class-III rapids received the highest partworth utility. A trip length of four hours was most preferred and eight hours was perceived negatively. The managerial attribute river access fee was most preferred in the level with $1.50 €$.

The social attribute number of people on the river received by far the highest relative importance (54.1\%). The resource attribute river difficulty was rated with the second highest relative importance $(14.5 \%)$, followed by the managerial attribute river access fee $(11.2 \%)$. The lowest rating was received by the social attribute waiting for parking $(4.6 \%)$.

\section{Discussion}

Study results revealed that more than two thirds of the boaters perceived crowding on the Salza River. All attributes tested were relevant to paddlers. However, one attribute, number of people on the river, elicited a very strong response from boaters. This information can be very useful for PA management to evaluate the importance boaters place on specific aspects of outdoor recreation experiences (Louviere \& Timmermans 1990).

\section{Boaters' crowding perceptions}

The river stretch between the towns of Wildalpen and Palfau is in some areas very narrow and therefore does not provide much space for large numbers of people. Our results of the crowding perception analysis confirm this speculation, as $70 \%$ of the boaters perceived crowding on the river, and boaters were only slightly less likely to be in sight of other groups than what they thought to be the acceptable limit. Therefore, there is potential that crowding might rise to concerning levels in the future (Shelby et al. 1989b). Comparing our crowding results with findings in other high-use settings (Kainzinger et al. 2016), we found that crowding in these high-use settings was slightly lower than in the Salza River data. An explanation for this difference could be that the high-use river used by Kainzinger et al. (2016) has a full allocation system, whereas on the Salza River only commercial rafting is regulated, and kayaking and canoeing are not limited to any group size. According to observations of the interviewers, crowding occurred specifically at the play spot next to the Wasserlochklamm (Figure 1). Here, congestion and crowding-related issues can be observed
Table 1 - Results of the choice model for the Salza River ( $n=324)$. The relative importance of each attribute on respondents' river choices is displayed in square brackets. ${ }^{*} p<.05,{ }^{* *} p<.01,{ }^{* * *} p<.001$

\begin{tabular}{|c|c|c|c|}
\hline \multicolumn{2}{|l|}{ Attributes } & Parameter & Std. Error \\
\hline \multirow[t]{19}{*}{ Social } & Number of people on the river & [54.1\%] & \\
\hline & 1 kayak and 1 person & 0.671 & \\
\hline & 4 kayaks and 4 people & ${ }^{* * *} 0.620$ & 0.085 \\
\hline & 1 raft and 6 people & ${ }^{* * *} 0.527$ & 0.081 \\
\hline & 8 kayaks and 8 people & ${ }^{* * *}-0.027$ & 0.054 \\
\hline & 12 kayaks and 12 people & -0.099 & 0.053 \\
\hline & 4 rafts and 24 people & ${ }^{* * *}-0.371$ & 0.074 \\
\hline & 6 kayaks, 6 rafts, 42 people & -0.604 & 0.068 \\
\hline & 8 rafts and 48 people & ${ }^{* * *}-0.717$ & 0.058 \\
\hline & Waiting time before launch & [9.0\%] & \\
\hline & $0 \mathrm{~min}$ & 0.204 & \\
\hline & $5 \mathrm{~min}$ & ${ }^{* * *} 0.176$ & 0.044 \\
\hline & $10 \mathrm{~min}$ & -0.004 & 0.044 \\
\hline & $20 \mathrm{~min}$ & $* *-0.376$ & 0.045 \\
\hline & Waiting time for parking & [4.6\%] & \\
\hline & $0 \mathrm{~min}$ & 0.121 & \\
\hline & $5 \mathrm{~min}$ & 0.063 & 0.040 \\
\hline & $10 \mathrm{~min}$ & -0.080 & 0.043 \\
\hline & $20 \mathrm{~min}$ & ${ }^{*}-0.104$ & 0.045 \\
\hline \multirow[t]{10}{*}{ Resource } & River difficulty & [14.5\%] & \\
\hline & 2 class-II rapids, no portage & -0.088 & \\
\hline & 2 class-III rapids, no portage & ${ }^{* * *} 0.221$ & 0.042 \\
\hline & 2 class-IV rapids, portage & ${ }^{* *}-0.107$ & 0.043 \\
\hline & 2 class-IV rapids, no portage & -0.027 & 0.044 \\
\hline & Trip length (time on the river) & {$[6.7 \%]$} & \\
\hline & $2 \mathrm{hrs}$ & 0.128 & \\
\hline & $4 \mathrm{hrs}$ & ${ }^{* * *} 0.153$ & 0.044 \\
\hline & $6 \mathrm{hrs}$ & 0.017 & 0.040 \\
\hline & $8 \mathrm{hrs}$ & $* * *-0.298$ & 0.040 \\
\hline \multirow[t]{5}{*}{ Managerial } & River access fee & [11.2\%] & \\
\hline & $1.50 €$ & 0.253 & \\
\hline & $3.00 €$ & -0.038 & 0.048 \\
\hline & $4.50 €$ & 0.006 & 0.048 \\
\hline & $6.00 €$ & ${ }^{* * *}-0.222$ & 0.044 \\
\hline
\end{tabular}

multiple times a day on a busy weekend (Figure 3). Without further regulations, there is potential for satisfaction to decrease and for negative impact on the resource.

\section{Boaters' preferences for social attributes}

Consistent with previous research in whitewater boating in North America, paddlers considered the social attribute number of people on the river as the most important one and preferred fewer people on the river (Kainzinger et al. 2016; Shelby \& Heberlein 1986; Stewart et al. 2003; Tarrant et al. 1997). Preferences for the number of people on the river showed a similar pattern to previous findings in high-use river settings. The most-preferred level was one person on the river, and boaters' preferences decreased with increasing riveruser numbers. This suggests that recreational paddlers in a high-use setting would prefer fewer people, but appear to tolerate the presence of other groups to a greater degree than boaters in low-use settings (Kainzinger et al. 2016). 
The waiting time for boat launching played a significant role for boaters. No waiting time was most preferred; waiting times of up to 5 minutes were acceptable; waiting 20 of minutes was disliked. These findings align with previous research suggesting that waiting times up to 5 minutes are acceptable for whitewater boaters in day-use settings (Kainzinger et al. 2016). Boaters on the Salza River might also have been worried about losing valuable river time at the boat ramp (Whittaker \& Shelby 1988).

Waiting time for parking was rated as having the least relative importance, and a waiting time longer than 5 minutes was disliked. This confirms that boaters seem to be more concerned about on-river congestion than facility-related issues (Kainzinger et al. 2016). Boaters on the Salza reported only minimal waiting times at the boat ramp or for parking. Even though parking opportunities are rare at the Salza River, waiting times to find a parking spot do not appear to be an issue for visitors and, consequently, are currently not of concern for the area management.

\section{Boaters' preferences for resource attributes}

Boaters on the Salza most preferred a river difficulty of class-III rapids, suggesting that they also have other motives than a challenge-driven experience. A recent study (Kainzinger et al. 2016) showed that whitewater boaters at two U.S. rivers with class-IV difficulty most preferred class-IV rapids with no portage, and saw themselves as capable of running at least a classIV rapid. Our results confirm that whitewater paddlers choose a specific setting based on their preferences for resource attributes, as the Salza is a relatively easy river, with only one class-III rapid. The majority of the Salza boaters rated their whitewater boating skills as intermediate and capable of running a class-III rapid at least.

The most preferred trip length was four hours, confirming past findings (Kainzinger et al. 2016). This attribute was rated with low relative importance, suggesting that trip length is not very relevant to whitewater boaters on the Salza. Consequently, Salza paddlers are looking for a whitewater experience with easy rapids that does not require highly specialized whitewater skills.

Boaters' preferences for managerial attributes

The managerial attribute was rated third in relative importance, suggesting that boaters on the Salza care to some extent about a river access fee. These boaters might be affected by the introduction of an access fee, fearing this would decrease the frequency of their participation in the whitewater activity (Kainzinger et al. 2016). Salza boaters would be willing to tolerate a river access fee of $1.50 €$ for a day trip. A high access fee might result in a decrease in the number of visits to the Nature Park, as some boaters might prefer to go to other rivers. However, an access fee might reduce crowding issues and resource impacts.
Boaters' trade-offs among social, resource and managerial attributes

This study found trade-off behaviour among social, resource and managerial attributes (Bullock \& Lawson 2008; Van Riper et al. 2011). In contrast to previous findings at high-use settings (Kainzinger et al. 2016), however, Salza paddlers rated the number of people on the river with much greater importance than the resource attribute river difficulty. This suggests that use levels play a significant role for Salza boaters. Kainzinger et al. (2016) examined a sample of more specialized and more committed boaters, who were mainly kayakers in a high-use setting. They had different motives and were looking for more challenge-driven experiences (Galloway 2012). Further research is necessary to explore how motives differ among boaters recreating in a high- and low-use river settings.

\section{Conclusions}

Using a multivariate approach, this study explored whitewater boaters' preferences for social, resource and managerial attributes in a high-use setting in Austria. The attribute number of people on the river was very important for boaters and plays a significant role in whitewater recreation; the high numbers of boaters with perceptions of crowding confirm that use-levels are an issue for the Salza River management. Further research should investigate whether paddlers are already employing coping behaviours due to crowded conditions.

Our results suggest that it might be useful to improve the current river-use regulation by regulating group sizes for kayaking and canoeing in addition to rafting. An allocation system that spreads the use throughout the day via an hourly launch limit system, with alternating periods of commercial and private users, may solve potential carrying-capacity issues. We also suggest the implementation of a visitor management framework such as VERP or LAC (National Park Service 1997; Stankey et al. 1985). Indicators and standards of the recreation experience and resource impacts should be integrated into these frameworks in order to build a basis for the permanent monitoring of use conditions and to regulate visitor use of the river in a participatory way.

The high use levels may be a threat for the Salza River ecosystem. River management in PAs often makes use of environmental education and interpretive signage to limit the impact of recreational use (Chiari 2010; Manning \& Anderson 2012). Such infrastructure elements could help attract boaters' attention to the valuable ecosystem of the Salza River and limit the impacts of the whitewater recreational use of the river.

Aukerman and Haas (2004) recommend that managers identify the recreational role of the resource in a specific area. The findings of our study have implications for the recreational management of the Alpine 
Nature and Geopark Styrian Eisenwurzen, who could use the information that the Salza attracts moderately experienced boaters to its advantage, by specifically targeting this group of boaters.

Further research on whitewater boating could also integrate attributes related to resource impacts due to recreational use (on, for example, gravel banks) in order to find a better balance between recreational use and ecological impacts in PAs.

\section{Acknowledgements}

We thank Jennifer Schöner for her assistance in developing the visual riverscape scenarios and Birgit Wallner for her help with the data collection.

\section{References}

Anderson, M., D. Kerstetter \& A.J. Graefe 1998. The effects of festival attributes upon perceptions of crowding. In: Vogelsong, H.G. (Comp., Ed.), Proceedings of the 1997 Northeastern Recreation Research Symposium (Gen. Tech. Rep. NE-241): 182-185.

Arnberger, A. \& R. Eder 2012. Exploring coping behaviours of Sunday and workday visitors due to dense use conditions in an urban forest. Urban Forestry \& Urban Greening 11(4): 439-449.

Arnberger, A. \& R. Eder 2015. Are urban visitors' general preferences for green-spaces similar to their preferences when seeking stress relief? Urban Forestry \& Urban Greening 14(4): 872-882.

Arnberger, A. \& W. Haider 2005. Social effects on crowding preferences of urban forest visitors. Urban Forestry \& Urban Greening 3(3): 125-136.

Arnberger, A. \& C. Mann 2008. Crowding in European forests: A review of recent research and implications for forest management and policy. Forestry 81(4): 559-571.

Aukerman, R. \& G.S. Haas 2004. Water recreation opportunity spectrum (WROS) users' guidebook. U.S. Department of Interior, Bureau of Reclamation, Colorado.

Bullock, S.D. \& S.R. Lawson 2008. Managing the "commons" on Cadillac Mountain: A stated choice analysis of Acadia National Park visitor's preferences. Leisure Sciences 30(1): 71-86.

Bricker, K.S. \& D.L. Kerstetter 2000. Level of specialization and place attachment: An exploratory study of whitewater recreationists. Leisure Sciences 22(4): 233-257.

Chiari, S. 2010. Raumbedarf für multifunktionale Flusslandschaften: Potentielle Synergien zwischen ökologischen Erfordernissen und Bedürfnissen der Freizeit- und Erholungsnutzung. Unpublished doctoral dissertation. University of Natural Resources and Life Sciences, Vienna, Austria. [In German]

Chiari, S., F. Schmid, A. Muhar \& S. Muhar 2008. Recreational functions of rivers in Austria: An approach to the visitors' perspective. In: Raschi, A. \& S. Trampetti, Management for protection and sustainable development. The Fourth International Conference on Monitoring and Management of Visitor Flow in Recreational and Protected Areas, Montecantini Terme.

Clark, R.N. \& G.H. Stankey 1979. The Recreation Opportunity Spectrum: A framework for planning, management and research. General Technical Report PNW-98. USDA Forest Service, Pacific Northwest Forest and Range Experiment Station.

Ditton, R.B., A.J. Fedler \& A.R. Graefe 1983. Factors contributing to perceptions of recreational crowding. Leisure Sciences 5(4): 273-288.

Galloway, S. 2012. Recreation specialization among New Zealand River recreation users: A multiactivity study of motivation and site preference. Leisure Sciences 34(3): 256-271.

Grand Canyon National Park 2006. Grand Canyon Colorado River Management Plan. U.S. Department of the Interior. Available at: https://www.nps.gov/ grca/learn/management/upload/CRMPIF_s.pdf (accessed on 01/18/2017)

Heberlein, T.A., J.P. Trent \& R.M. Baumgartner 1982. The influence of hunter density on firearm deer hunters' satisfaction. Transactions of the 47 th North American Wildlife and Natural Resources Conference 47: 665-676.

Heberlein, T.A. \& J.J. Vaske 1977. Crowding and visitor conflict on the Bois Brule River (report WISC WRC 77-04). Madison, WI: University of Wisconsin Water Resources Center.

Hensher, D.A., J.M. Rose \& W.H. Greene 2005. Applied choice analysis: A primer.

Herrick, T.A. \& C.D. McDonald 1992. Factors affecting overall satisfaction with a river recreation experience. Environmental Management 16(2): 243-247.

Kainzinger, S., A. Arnberger \& R.C. Burns 2016. Setting preferences of high and low use river recreationists: how different are they? Environmental Management 58(5): 767-779.

Lange, E. 2001. The limits of realism: Perceptions of virtual landscapes. Landscape and Urban Planning 54(1-4): 163-182.

Lawson, S.R. \& R.E. Manning 2002. Tradeoffs among social, resource, and management attributes of the Denali wilderness experience: A contextual approach to normative research. Leisure Sciences 24(3-4): 297-312.

Lee, S., A. Graefe \& C. Li 2007. The effect of specialization and gender on motivations and preferences for site attributes in paddling. Leisure Sciences 29(4): 355-373.

Louviere, J.J., D.A. Hensher \& J. Swait 2000. Stated choice methods: Analysis and application. Cambridge, UK.

Louviere, J. \& H. Timmermans 1990. Stated preference and choice models applied to recreation research: A review. Leisure Sciences 12(1): 9-32.

Manning, R.E. 2011. Studies in outdoor recreation: Search and research for satisfaction $\left(3^{\text {rd }} \mathrm{ed}\right.$.). Corvallis, OR.

Manning, R.E. 2013. Parks and carrying capacity: Commons without tragedy. 
Manning, R.E. \& L.E. Anderson 2012. Managing outdoor recreation: Case studies in the National Parks. Oxfordshire, UK.

Manning, R.E., D.W. Lime, M. Hof, \& W.A. Freimund 1995. The Visitor Experience and Resource Protection (VERP) process: The application of carrying capacity to Arches National Park. Georg Wright Forum 12: 41-55.

National Park Service. 1997. The visitor experience and resource protection framework: $A$ bandbook for planners and managers. US Department of the Interior, National Park Service, Denver Service Center, Denver, CO.

Palmer, J.F. \& R.E. Hoffman 2001. Rating reliability and representation validity in scenic landscape assessments. Landscape and Urban Planning 54(1-4): 149-161.

Raftingverband Steiermark 2016. Available at http://www.raftingverband.at/ (accessed on 10/08/2016) [In German]

Reichhart, T. \& A. Arnberger 2010. Exploring the influence of speed, social, managerial, and physical factors on shared trail preferences using a 3D computer animated choice experiment. Landscape and Urban Planning 96(1): 1-11.

Schuett, M.A. 1993. Refining measures of adventure recreation involvement. Leisure Sciences 15(3): 205-216.

Shelby, B. \& T.A. Heberlein 1986. Carrying capacity in recreation settings. Corvallis, $\mathrm{OR}$.

Shelby, B., D. Whittaker \& M. Danley.1989a. Idealism versus pragmatism in user evaluations of allocation systems. Leisure Sciences 11(1): 61-70.

Shelby, B, J. Vaske \& T. Heberlein 1989b. Comparative analysis of crowding in multiple locations: Results from fifteen years of research. Leisure Science 11(4): 269-291.

Siderelis, C. \& R.L. Moore 2006. Examining the effects of hypothetical modifications in permitting procedures and river conditions on whitewater boating behavior. Journal of Leisure Research 38(4): 558-574.

Stankey, G.H., D.N. Cole, R.C. Lucas, M.E. Petersen \& S.S. Frissell 1985. The Limits of Acceptable Change (LAC) system for wilderness planning. United States DA Forest Service, General Technical Reports. Intermountain Forest and Range Experiment Station, Ogden, UT.

Stewart, W., K. Larkin, B. Orland \& D. Anderson 2003. Boater preferences for beach characteristics downstream from Glen Canyon Dam, Arizona. Journal of Environmental Management 69(2): 201-211.

Stewart, W., K. Larkin, B. Orland, N. Tomar, D. Anderson, R. Manning, D. Cole \& J. Taylor 2000. Preferences of recreation user groups of the Colorado River in Grand Canyon. University of Illinois.

Tarrant, M.A., H.K. Cordell \& T.L. Kibler 1997. Measuring perceived crowding for high-density river recreation: The effects of situational conditions and personal factors. Leisure Sciences 19(2): 97-112.

Van Riper, C.J., R.E. Manning, C.A. Monz \& K.A. Goonan 2011. Tradeoffs among resource, social, and managerial conditions on mountain summits of the Northern Forest. Leisure Sciences 33(3): 228-249.

Vaske, J.J. 2008. Survey research and analysis: Applications in parks, recreation and buman dimensions. State College, PA.

Vaske, J.J. \& L.B. Shelby 2008. Crowding as a descriptive indicator and an evaluative standard: Results form 30 years of research. Leisure Sciences 30(2): 111126.

Vermunt, J.K. \& J. Magidson 2003. Latent Gold Choice user's manual. Statistical Innovations, Inc.

Whisman, S.A. \& S.J. Hollenhorst 1998. A path model of whitewater boating satisfaction on the Cheat River of West Virginia. Environmental Management 22(1): 109-117.

Whittaker, D. \& B. Shelby 2008. Allocating river use: A review of approaches and existing systems for river professionals. River Management Society.

Whittaker, D. \& B. Shelby 1988. Types of norms for recreation impacts: Extending the social norms concept. Journal of Leisure research 20(4): 261-273.

\section{Authors}

\section{Silvia Kainzinger ${ }^{1,2}$}

studied Landscape Planning and Landscape Architecture at the University of Natural Resources and Life Sciences (Boku), in Vienna, Austria. Her research focuses on the recreational preferences and recreation specializations of whitewater kayakers and rafters. She works as a graduate assistant, alternating between Austria and the United States. Peter Jordan-Strasse 82, 1190 Vienna, Austria. Email: silvia.kainzinger@boku.ac.at

\section{Arne Arnberger ${ }^{1}$}

is an Associate Professor. His research focuses on eco- and rural tourism, outdoor recreation, visitor monitoring, landscape and human health, protected area management, landscape aesthetics, and biosphere reserves.

\section{Robert Burns ${ }^{2}$}

is Director and Professor at West Virginia University's Division of Forestry and Natural Resources. He teaches classes related to outdoor recreation, integrating international research efforts into his teaching. He has published several international articles comparing US natural resource areas with those of central Europe and Brazil.

${ }^{1}$ Institute of Landscape Development, Recreation and Conservation Planning, Department of Spatial, Landscape and Infrastructure Sciences, University of Natural Resources and Life Sciences, Vienna, Austria.

${ }^{2}$ Recreation, Parks and Tourism Program, Division of Forestry and Natural Resources, West Virginia University. 